\title{
Bi-Lipschitz embeddings of hyperspaces of compact sets
}

\author{
by
}

Jeremy T. Tyson (Urbana, IL)

\begin{abstract}
We study the bi-Lipschitz embedding problem for metric compacta hyperspaces. We observe that the compacta hyperspace $K(X)$ of any separable, uniformly disconnected metric space $X$ admits a bi-Lipschitz embedding in $\ell^{2}$. If $X$ is a countable compact metric space containing at most $n$ nonisolated points, there is a Lipschitz embedding of $K(X)$ in $\mathbb{R}^{n+1}$; in the presence of an additional convergence condition, this embedding may be chosen to be bi-Lipschitz. By way of contrast, the hyperspace $K([0,1])$ of the unit interval contains a bi-Lipschitz copy of a certain self-similar doubling seriesparallel graph studied by Laakso, Lang-Plaut, and Lee-Mendel-Naor, and consequently admits no bi-Lipschitz embedding into any uniformly convex Banach space. Schori and West proved that $K([0,1])$ is homeomorphic with the Hilbert cube, while Hohti showed that $K([0,1])$ is not bi-Lipschitz equivalent with a variety of metric Hilbert cubes.
\end{abstract}

1. Introduction. The topological structure of hyperspaces has been intensively studied during the twentieth century. According to a celebrated result of Curtis and Schori [7], the hyperspace of any nondegenerate Peano continuum is homeomorphic with the Hilbert cube. An essential reference for the topology of hyperspaces is the book [22] by Illanes and Nadler.

Hyperspaces of metric spaces are naturally metrized by the Hausdorff metric. They play a role in dynamical systems, as the existence of attractors for iterated function systems on complete metric spaces may be demonstrated by an application of a suitable fixed point theorem to the hyperspace. This approach to fractal geometry was first presented by Hutchinson [21] (but see Moran [32] for earlier ideas along the same lines) and is now standard.

Motivated by these and other applications, some recent studies have focused on the intrinsic metric geometry of hyperspaces. Boardman, Goodey and McClure [5], [13], [14], [31] studied the generalized Hausdorff measures

2000 Mathematics Subject Classification: Primary 54B20; Secondary 05C10, 26A16, 26A18, 28A80, 46B20.

Key words and phrases: compacta hyperspace, bi-Lipschitz embedding, iterated function system, series-parallel graph, round ball metric space.

Supported by the National Science Foundation under Award No. DMS-0228807. 
of hyperspaces of self-similar sets, while Hohti [20] showed that the hyperspace of the closed unit interval is not bi-Lipschitz equivalent with a variety of metric Hilbert cubes. Gruber, Lettl and Tichy [15], [16], [17] investigated the isometries of Euclidean and general hyperspaces. See also Bandt [3].

In this paper we study the existence of bi-Lipschitz embeddings of compacta hyperspaces in specified targets. We observe that the hyperspace of any separable, uniformly disconnected metric space admits a bi-Lipschitz embedding in $\ell^{2}$. We prove that the hyperspace of any countable compact metric space containing at most $n$ nonisolated points admits a Lipschitz embedding in $\mathbb{R}^{n+1}$; provided a further convergence criterion is satisfied, the embedding in question is bi-Lipschitz.

On the other hand, we show that the hyperspace $K([0,1])$ of the closed unit interval admits no bi-Lipschitz embedding in any uniformly convex Banach space, and more generally, it admits no bi-Lipschitz embedding in any round ball metric space, a concept introduced by Laakso [26]. Note that $K([0,1])$ is topologically equivalent with the Hilbert cube, as was shown by Schori and West [37], [38]. To give another proof of this result, we exhibit within $K([0,1])$ a bi-Lipschitz copy of a certain series-parallel graph $G$ studied by Laakso [26], Lang-Plaut [27] and Lee-Mendel-Naor [28]. Laakso [26] showed that a variant of $G$ admits no bi-Lipschitz embedding in any round ball metric space; his argument can be adapted to apply to $G$. We construct a planar iterated function complex (generalized iterated function system) parameterized by an uncountable collection of geodesic segments in $G$. The desired embedding of $G$ in $K([0,1])$ is obtained by choosing appropriate vertical slices of the attractors for this complex.

For recent results on the bi-Lipschitz and isometric embedding problem into hyperspaces of closed unbounded sets, as well as other results on the metric geometry of hyperspaces, see [24].

In Section 2, we review some standard topics in metric geometry, give further history and motivation, and state our main results. In Section 3 we consider countable compact metric spaces and finite-dimensional Euclidean targets, while Section 4 contains the proof of the nonembeddability of the hyperspace of the unit interval in any round ball metric space. A concluding Section 5 contains some questions for future study.

Acknowledgements. We are grateful to the referee for a careful reading of the paper.

\section{Review of metric geometry and main results}

2.1. Notation and basic definitions. For a metric space $X=(X, d)$, we write $\operatorname{diam} A$ (or $\operatorname{diam}_{d} A$ in case the metric deserves to be mentioned) for the diameter of a set $A \subset X$ and $\operatorname{dist}(A, B)$ for the distance between two 
nonempty sets $A, B \subset X$. We abbreviate $\operatorname{dist}(A, x)=\operatorname{dist}(A,\{x\})$ for $A \subset X$ and $x \in X$. We denote by $B(x, r)$ the closed ball in $X$ with center $x$ and radius $r$. A metric space $(X, d)$ is doubling if there exists a finite constant $M$ so that every ball $B(x, r)$ in $X$ can be covered by at most $M$ balls of radius $r / 2$.

A modulus of continuity for a map $f:(X, d) \rightarrow\left(Y, d^{\prime}\right)$ between compact metric spaces is an increasing function $\omega:[0, \operatorname{diam} X] \rightarrow[0, \infty)$ satisfying

$$
d^{\prime}(f(x), f(y)) \leq \omega(d(x, y))
$$

for all $x, y \in X$. The map $f$ is L-Lipschitz, $L<\infty$, if it has a modulus of continuity of the form $\omega(t)=L t$, and is $\beta$-Hölder continuous, $0<\beta \leq 1$, if it has a modulus of continuity of the form $\omega(t)=C t^{\beta}$ for some $C<\infty$. If the values of the constants $L$ or $\beta$ are not important, the terms Lipschitz or Hölder continuous are used. An $L$-Lipschitz map with $L<1$ is called a contraction.

If $f$ is a homeomorphism and $f$ and $f^{-1}$ are both $(L$-)Lipschitz, $f$ is said to be (L-)bi-Lipschitz. If $f^{-1}$ is Hölder, $f$ is said to be co-Hölder.

A gauge function is a nondecreasing, right-continuous function $\varphi:[0, \infty)$ $\rightarrow[0, \infty)$ with $\varphi(0)=0$. Associated with each gauge function is the generalized Hausdorff $\varphi$-measure $\mathcal{H}^{\varphi}$ on $X$, defined for $E \subset X$ as

$$
\mathcal{H}^{\varphi}(E):=\liminf _{\delta \rightarrow 0}\left\{\sum_{i} \varphi\left(\operatorname{diam} E_{i}\right): \operatorname{diam} E_{i} \leq \delta \text { for all } i \in \mathbb{N}, X=\bigcup_{i} E_{i}\right\} .
$$

The case $\varphi_{s}(t)=t^{s}$ gives the classical Hausdorff measures $\mathcal{H}^{s}:=\mathcal{H}^{\varphi_{s}}$.

We write $s \vee t$, resp. $s \wedge t$, for the maximum, resp. minimum, of two real numbers $s$ and $t$.

2.2. Hyperspaces and the Hausdorff metric. The hyperspace of compact sets (or (compacta) hyperspace) of a topological space $X$ is the space $K(X)$ of all nonempty compact subsets of $X$. When $X=(X, d)$ is a metric space, $K(X)$ is equipped with the Hausdorff metric

$$
\begin{aligned}
D(A, B)=D_{d}(A, B) & :=\inf \left\{\varepsilon: A \subset N_{\varepsilon}(B) \text { and } B \subset N_{\varepsilon}(A)\right\} \\
& =\max _{a \in A} \operatorname{dist}(a, B) \vee \max _{b \in B} \operatorname{dist}(b, A)
\end{aligned}
$$

for $A, B \in K(X)$, where $N_{\varepsilon}(A)=\{x \in X: d(x, a)<\varepsilon$ for some $a \in A\}$ denotes the $\varepsilon$-neighborhood of $A$.

The hyperspace $K(X)$ of a complete (resp. compact) space $X$ is complete (resp. compact). See, e.g., [22, Chapter I]. Furthermore, $X$ embeds isometrically in $K(X)$ and $\operatorname{diam}_{D} K(X)=\operatorname{diam}_{d} X$. The map $A \mapsto \operatorname{diam} A$ is a 2-Lipschitz map from $K(X)$ to $\mathbb{R}$. If $X$ is separable, then $K(X)$ is also separable. Indeed, if $Y$ is a countable dense subset of $X$, then the collection of all finite subsets of $Y$ is a countable dense subset of $K(X)$. 
2.3. Hyperspaces of disconnected spaces. A metric $d$ on a space $X$ is called an ultrametric if $d(x, y) \leq \max \{d(x, z), d(z, y)\}$ for all $x, y, z \in X$. Compacta hyperspaces of ultrametric spaces embed isometrically in Hilbert spaces.

2.4. Proposition. Let $(X, d)$ be an ultrametric space. Then $K(X)$ admits an isometric embedding in a Hilbert space.

The solution to the isometric embedding problem for Hilbert space targets was a major advance in twentieth century metric geometry. Work of Schoenberg [35], [36] and Blumenthal [4] provided an intrinsic characterization of metric spaces admitting an isometric embedding in a Hilbert space. Timan [39], Lemin [29] and Aschbacher et al. [1] gave independent proofs that every ultrametric space admits an isometric embedding in a Hilbert space. Since hyperspaces of ultrametric spaces are ultrametric, Proposition 2.4 follows.

A metric space $(X, d)$ is called uniformly disconnected if it is bi-Lipschitz equivalent with an ultrametric space. Uniformly disconnected spaces were introduced by David and Semmes [9, Chapter 15] using a different but equivalent definition.

2.5. Corollary. Let $(X, d)$ be a uniformly disconnected space. Then $K(X)$ admits a bi-Lipschitz embedding in a Hilbert space.

This is an immediate consequence of Proposition 2.4, together with the observation that each bi-Lipschitz map $f: X \rightarrow Y$ induces a bi-Lipschitz $\operatorname{map} F_{f}: K(X) \rightarrow K(Y)$ by $F_{f}(A)=\{f(a): a \in A\}$.

Since hyperspaces of separable spaces are separable, and separable ultrametric spaces embed isometrically in $\ell^{2}$, we also have the following

2.6. Corollary. If $(X, d)$ is separable and uniformly disconnected, then $K(X)$ admits a bi-Lipschitz embedding in $\ell^{2}$.

For example, the hyperspace of the standard Cantor set $C$ admits a bi-Lipschitz embedding in $\ell^{2}$. Observe that $K(C)$ is perfect and totally disconnected, hence homeomorphic with $C$. We do not know to what extent this topological equivalence can be made quantitative.

2.7. QuESTION. What is the optimal modulus of continuity for a homeomorphism of $K(C)$ onto $C$ ?

The characterization of metric spaces which admit a bi-Lipschitz embedding in a finite-dimensional Euclidean space is a major open problem in geometric analysis. The following theorem provides some infinite metric spaces whose hyperspaces admit such an embedding. 
2.8. TheOREM. Let $X$ be a compact metric space containing at most $N$ nonisolated points. Let $I(X)=\left\{x_{1}, x_{2}, \ldots\right\}$ be an enumeration of the isolated points of $X$ so that the sequence $d_{n}:=\operatorname{dist}\left(x_{n}, X \backslash I(X)\right)$ is nonincreasing. Then

(a) there exists a Lipschitz embedding of $K(X)$ in $\mathbb{R}^{N+1}$,

(b) if

$$
r^{+}:=\limsup _{n \rightarrow \infty} \frac{d_{n+1}}{d_{n}}<1
$$

then the embedding in part (a) may be chosen to be co-Hölder,

(c) if $r^{+}<1 / 2$, then the embedding in part (a) may be chosen to be bi-Lipschitz.

For example, the hyperspace of $X=\left\{a^{n}: n=1,2, \ldots\right\} \cup\{0\}, a<1$, admits an embedding in $\mathbb{R}^{2}$ which is Lipschitz and co-Hölder. When $a<1 / 2$ the embedding is bi-Lipschitz.

Pełczyński [33] characterized the hyperspaces of compact totally disconnected topological spaces containing a dense set of isolated points: for every such space $X$, the hyperspace $K(X)$ is homeomorphic with the union of the Cantor set and the midpoints of the omitted intervals. Metric analogues of this result of Pełczyński remain to be established.

Observe that a condition such as (2.9) is necessary for the conclusion in Theorem 2.8. Indeed, for any prescribed Hausdorff gauge function $\varphi$ there exists a countable metric space $(X, d)$ with precisely one nonisolated point, so that the generalized Hausdorff measure $\mathcal{H}^{\varphi}(K(X))$ is positive and finite. See, for example, Theorem 3.5 in [31].

We will prove our Theorem 2.8 in Section 3.

2.10. The hyperspace of the unit interval. Characterizing the hyperspace of $[0,1]$ was a long-standing problem in the topology of hyperspaces. In 1938, Wojdysławski conjectured that $K([0,1])$ was homeomorphic to the Hilbert cube $Q=[0,1]^{\infty}$. The conjecture stood for over thirty years, until Schori and West proved it in 1975 [38].

The metric structure of $K([0,1])$ was studied by Boardman and Goodey, who determined some gauge functions $\varphi$ with respect to which $K([0,1])$ has zero or infinite generalized Hausdorff measure [13], [14], [5]. The results of Boardman and Goodey were extended to the setting of general self-similar sets satisfying the open set condition by McClure [31].

A metric space $X$ is called bi-Lipschitz homogeneous if the group of biLipschitz self-homeomorphisms of $X$ acts transitively. In response to a question posed by Väisälä [41], Hohti [20] showed that the metric Hilbert cube $\left(Q, d_{s}\right) \subset c_{0}$ is bi-Lipschitz homogeneous if and only if

$$
\sup _{k} \frac{s_{k}}{s_{k+1}}<\infty
$$


Here $s=\left(s_{k}\right)_{k \in \mathbb{N}}$ is a decreasing sequence of positive real numbers converging to zero, and $d_{s}(x, y)=\max _{k} s_{k}\left|x_{k}-y_{k}\right|$ for $x=\left(x_{k}\right), y=\left(y_{k}\right) \in Q$. On the other hand, Hohti also showed in $[20]$ that $K([0,1])$ is not bi-Lipschitz homogeneous. It follows that $K([0,1])$ is not bi-Lipschitz equivalent with $\left(Q, d_{s}\right)$ for any $s$ satisfying $(2.11)$.

Using a comparison principle for the cardinality of nets in bi-Lipschitz equivalent spaces, Hohti also demonstrated the bi-Lipschitz inequivalence of $K([0,1])$ with $(Q, d)$ for some other metrics $d$. For example, $K([0,1])$ and $\left(Q, \varrho_{s}\right) \subset \ell^{1}$ are bi-Lipschitz inequivalent, where $s=\left(s_{k}\right), s_{k}=2^{-k}$, and $\varrho_{s}(x, y)=\sum_{k} s_{k}\left|x_{k}-y_{k}\right|$. The proof easily extends to the case $\left(Q, \varrho_{s, p}\right) \subset \ell^{p}$, $1<p<\infty$, where $\varrho_{s, p}(x, y)=\left(\sum_{k} s_{k}^{p}\left|x_{k}-y_{k}\right|^{p}\right)^{1 / p}$.

The following theorem further illustrates the intrinsic metric complexity of $K([0,1])$ and significantly extends the preceding results of Hohti.

2.12. TheOREM. There is no bi-Lipschitz embedding of $K([0,1])$ into any round ball metric space.

Following Laakso [26], we say that a metric space $(X, d)$ is a round ball space if for every $\varepsilon>0$ there exists $\delta=\delta(\varepsilon)>0$ so that

$$
\operatorname{diam} B(x,(1 / 2+\delta) d(x, y)) \cap B(y,(1 / 2+\delta) d(x, y)) \leq \varepsilon d(x, y)
$$

for all $x, y \in X$. We call $\delta(\varepsilon)$ the roundness function of $X$. If $X$ is a Banach space, then $X$ is a round ball space if and only if $X$ is uniformly convex [26]. Bi-Lipschitz embeddings in round ball metric spaces were studied by Laakso [26] in connection with strong $A_{\infty}$ deformations of geometry and by Tyson and $\mathrm{Wu}[40]$ in connection with snowflake metric spaces and Assouad's embedding theorem [2].

With no a priori control on the geometry of the target, it is not clear whether one can prove Theorem 2.12 by the techniques of [20]. Note also that generalized Hausdorff measures cannot be used to rule out the existence of such an embedding. Indeed, a classical result of Goodey [12] asserts that every infinite-dimensional Banach space has non- $\sigma$-finite Hausdorff measure for every gauge function $\varphi$.

To prove Theorem 2.12, we use recent results on the bi-Lipschitz nonembeddability of certain series-parallel graphs due to Laakso, Lang-Plaut and Lee-Mendel-Naor. Consider the infinite graph $G$ obtained as the Gromov-Hausdorff limit of the sequence of finite series-parallel graphs $G_{n}$ shown in Figure 1. In rough terms, $G_{n}$ is obtained by replacing each edge in $G_{n-1}$ with a scaled copy of the graph $G_{1}$. For the precise definition, see Definition 4.8. We equip $G$ with the path metric.

Laakso [26] used a variant of this graph to answer a question of Heinonen and Semmes [19, Question 1] on the existence of a planar strong $A_{\infty}$ weight (in the sense of David and Semmes [8], [9]) whose deformed geometry fails 


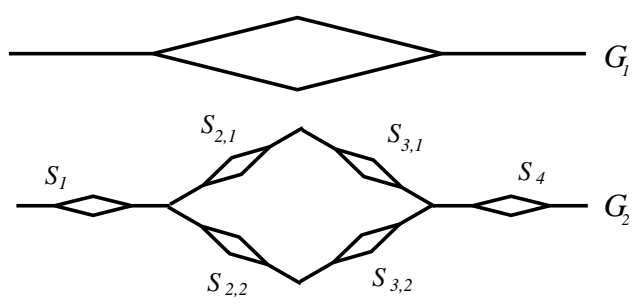

Fig. 1. Approximations of the Laakso graph $G$

to admit a bi-Lipschitz embedding in any uniformly convex Banach space. Motivated by algorithmic problems in network theory and the failure of the Johnson-Lindenstrauss dimension reduction lemma in $L^{1}$, Lee, Mendel and Naor [28] showed that $G$ admits no bi-Lipschitz embedding in $L^{p}$ for any $1<p \leq 2$. See also Lang and Plaut [27]. Following the terminology of [28], we call $G$ the Laakso graph.

2.14. Proposition. The Laakso graph $G$ does not admit a bi-Lipschitz embedding in any round ball metric space.

For the sake of completeness, we include a proof of Proposition 2.14 in Section 4, following the argument given by Laakso [26, §2.5].

Theorem 2.12 is a consequence of Proposition 2.14 and the following theorem, which we also prove in Section 4.

2.15. THEOREM. There is a bi-Lipschitz embedding of the Laakso graph $G$ in $K([0,1])$.

We do not know whether $K([0,1])$ admits a bi-Lipschitz embedding in $L^{1}$, or whether every bounded series-parallel graph admits a bi-Lipschitz embedding in $K([0,1])$. Gupta et al. $[18]$ have shown that $\ell^{1}$ contains a 14 -biLipschitz copy of every series-parallel graph.

For additional questions and discussion, see Section 5 .

3. Proof of Theorem 2.8. Throughout this section, we let $X$ be a compact metric space and we denote by $I(X)$, resp. $L(X)$, the set of isolated, resp. limit, points of $X$. As in the statement of Theorem 2.8, we assume that $I(X)=\left\{x_{1}, x_{2}, \ldots\right\}$, where $d_{n}:=\operatorname{dist}\left(x_{n}, L(X)\right)$ is nonincreasing. For $x \in I(X)$, we define the gap of $X$ at $x$ to be

$$
\operatorname{gap}(X, x):=\inf \{d(x, y): y \in X, x \neq y\} .
$$

Fix $\lambda>2$ and define

$$
\gamma_{n}:=\min \left\{\lambda^{i-n} \operatorname{gap}\left(X, x_{i}\right): i=1, \ldots, n\right\} .
$$

3.1. Lemma. For each $n \in \mathbb{N}$,

$$
\sum_{m>n} \gamma_{m} \leq \frac{1}{\lambda-1} \gamma_{n} .
$$


Proof. For each $i=1, \ldots, n$,

$$
\sum_{m>n} \gamma_{m} \leq \sum_{m=n+1}^{\infty} \lambda^{i-m} \operatorname{gap}\left(X, x_{i}\right)=\frac{1}{\lambda-1} \lambda^{i-n} \operatorname{gap}\left(X, x_{i}\right)
$$

and the result follows.

The following theorem gives a more precise statement of part (a) of Theorem 2.8 .

3.2. TheOREM. Let $X$ be a countable compact metric space with $N$ limit points. Then there is a Lipschitz embedding $F$ of $K(X)$ in $\mathbb{R}^{N+1}$ whose inverse has a modulus of continuity

$$
\omega(t)=t+2 \omega_{0}\left(\frac{\lambda-1}{\lambda-2} t\right)
$$

where $\omega_{0}$ is any increasing function from $[0, \infty)$ to $[0, \infty)$ with $\omega_{0}(0)=0$ and

$$
d_{n} \leq \omega_{0}\left(\gamma_{n}\right)
$$

for all $n$.

Proof. We denote points in $\mathbb{R}^{N+1}$ by $x=\left(x_{0}, \ldots, x_{N}\right)$, and use the maximum metric

$$
\|x-y\|_{\infty}=\max _{i=0, \ldots, M}\left|x_{i}-y_{i}\right|, \quad x, y \in \mathbb{R}^{N+1} .
$$

Let $L(X)=\left\{z_{1}, \ldots, z_{N}\right\}$ and define $F=\left(f_{0}, \ldots, f_{N}\right): K(X) \rightarrow \mathbb{R}^{N+1}$ by

$$
f_{i}(A)=\operatorname{dist}\left(A, z_{i}\right), \quad i=1, \ldots, N,
$$

and

$$
f_{0}(A)=\sum_{n: x_{n} \in A} \gamma_{n}
$$

Observe that $f_{0}(A)=f_{0}(A \cap I(X))$ for all $A \in K(X)$.

3.5. Lemma. Suppose that $A \cap I(X) \neq B \cap I(X)$ for some $A, B \in K(X)$. Then

$$
\frac{\lambda-2}{\lambda-1} \gamma_{n_{1}} \leq\left|f_{0}(A)-f_{0}(B)\right| \leq \frac{\lambda}{\lambda-1} \gamma_{n_{1}}<2 \gamma_{n_{1}},
$$

where $n_{1}=\min \left\{n: x_{n} \in A \triangle B\right\}$.

Here $A \triangle B:=(A \backslash B) \cup(B \backslash A)$ denotes the symmetric difference of $A$ and $B$.

Proof of Lemma 3.5. Without loss of generality assume that $x_{n_{1}} \in A$. By Lemma 3.1, 


$$
\begin{aligned}
\left|f_{0}(A)-f_{0}(B)\right| & =\left|\gamma_{n_{1}}+\sum_{n>n_{1}: x_{n} \in A \backslash B} \gamma_{n}-\sum_{n>n_{1}: x_{n} \in B \backslash A} \gamma_{n}\right| \\
& \geq \gamma_{n_{1}}-\sum_{n>n_{1}} \gamma_{n} \geq \frac{\lambda-2}{\lambda-1} \gamma_{n_{1}} .
\end{aligned}
$$

In a similar manner, we have $\left|f_{0}(A)-f_{0}(B)\right| \leq \gamma_{n_{1}}+\sum_{n>n_{1}} \gamma_{n} \leq \frac{\lambda}{\lambda-1} \gamma_{n_{1}}$.

Returning to the proof of Theorem 3.2, let $A, B \in K(X), A \neq B$. If $A \cap I(X)=B \cap I(X)$ then $f_{0}(A)=f_{0}(B)$. Otherwise, using Lemma 3.5 we find

$$
\left|f_{0}(A)-f_{0}(B)\right| \leq 2 \gamma_{n_{1}} \leq 2 \operatorname{gap}\left(X, x_{n_{1}}\right) \leq 2 D(A, B) .
$$

On the other hand, for any $i=1, \ldots, N$ and any $b \in B$,

$$
f_{i}(A)=\operatorname{dist}\left(A, z_{i}\right) \leq \operatorname{dist}(A, b)+d\left(b, z_{i}\right) \leq D(A, B)+d\left(b, z_{i}\right)
$$

so $f_{i}(A) \leq D(A, B)+f_{i}(B)$. Reversing the roles of $A$ and $B$ shows that

$$
\left|f_{i}(A)-f_{i}(B)\right| \leq D(A, B),
$$

and combining (3.7) and (3.8) yields

$$
\|F(A)-F(B)\|_{\infty} \leq 2 D(A, B) .
$$

To compute a modulus of continuity for $F^{-1}$, let $A, B \in K(X), A \neq B$. If $A \cap I(X)=B \cap I(X)$, then $A \cap L(X) \neq B \cap L(X)$ and

$D(A, B)=\max _{a \in A} \operatorname{dist}(a, B) \vee \max _{b \in B} \operatorname{dist}(b, A)=\max _{a \in A^{\prime}} \operatorname{dist}(a, B) \vee \max _{b \in B^{\prime}} \operatorname{dist}(b, A)$, where $A^{\prime}=(A \backslash B) \cap L(X)$ and $B^{\prime}=(B \backslash A) \cap L(X)$. (Note that $A^{\prime} \cup B^{\prime} \neq \emptyset$.) Thus

$$
\begin{aligned}
D(A, B) & \leq \max _{z_{i} \in A^{\prime} \cup B^{\prime}}\left|f_{i}(A)-f_{i}(B)\right| \leq\|F(A)-F(B)\|_{\infty} \\
& \leq \omega\left(\|F(A)-F(B)\|_{\infty}\right),
\end{aligned}
$$

where $\omega(t)$ is as in (3.3).

Suppose instead that $A \cap I(X) \neq B \cap I(X)$ and let $A^{\prime \prime}=(A \backslash B) \cap I(X)$ and $B^{\prime \prime}=(B \backslash A) \cap I(X)$. Choose $n_{1}$ as in Lemma 3.5. For each $a \in A^{\prime \prime}$, $\operatorname{dist}(a, L(X))=d\left(a, z_{i}\right)$ for some $z_{i} \in L(X)$. Hence $f_{i}(A) \leq d\left(a, z_{i}\right) \leq d_{n_{1}}$ and

$$
\begin{aligned}
\operatorname{dist}(a, B) & \leq d\left(a, z_{i}\right)+\operatorname{dist}\left(z_{i}, B\right) \leq d_{n_{1}}+f_{i}(B) \\
& \leq 2 d_{n_{1}}+\left|f_{i}(B)-f_{i}(A)\right| \leq 2 d_{n_{1}}+\|F(B)-F(A)\|_{\infty} .
\end{aligned}
$$


Similarly $\operatorname{dist}(b, A) \leq 2 d_{n_{1}}+\|F(B)-F(A)\|_{\infty}$ for all $b \in B^{\prime \prime}$. It follows that (3.11)

$$
\begin{aligned}
D(A, B) & =\max _{a \in A^{\prime \prime}} \operatorname{dist}(a, B) \vee \max _{b \in B^{\prime \prime}} \operatorname{dist}(b, A) \\
& \leq 2 d_{n_{1}}+\|F(B)-F(A)\|_{\infty} \\
& \leq 2 \omega_{0}\left(\gamma_{n_{1}}\right)+\|F(B)-F(A)\|_{\infty} \\
& \leq 2 \omega_{0}\left(\frac{\lambda-1}{\lambda-2}\left|f_{0}(B)-f_{0}(A)\right|\right)+\|F(B)-F(A)\|_{\infty} \\
& \leq \omega\left(\|F(B)-F(A)\|_{\infty}\right) .
\end{aligned}
$$

We used Lemma 3.5 in the penultimate step.

3.12. REMARK. The construction in the preceding proof was inspired by a paper of Reiter and Reiter [34], who gave an explicit form for the embedding in the theorem of Pełczyński in the setting of compact metric spaces with a single nonisolated point.

Proof of Theorem 2.8(b),(c). Assume that $r^{+}<1$ and choose $r^{+}<\varrho<1$. If $r^{+}<1 / 2$ choose $r^{+}<\varrho<1 / 2$. Then there exists $n_{2}$ so that $d_{n+1} \leq \varrho d_{n}$ for all $n \geq n_{2}$. Let $Z=\left\{x_{1}, \ldots, x_{n_{2}}\right\}$ and let

$$
\varepsilon_{Z}=\min _{x_{n} \in Z} \operatorname{gap}\left(X, x_{n}\right) \text {. }
$$

The Lipschitz/Hölder constant for $F^{-1}$ will depend on the parameters $n_{2}$ and $\varepsilon_{Z}$.

3.13. Lemma. For all $n>n_{2}, \operatorname{gap}\left(X, x_{n}\right) \geq(1-\varrho) d_{n}$.

Proof. For $n \in \mathbb{N}$,

$$
\begin{aligned}
\operatorname{gap}\left(X, x_{n}\right) & =\inf \left\{d\left(x_{i}, x_{n}\right): i \neq n\right\} \\
& \geq\left(d_{1}-d_{n}\right) \wedge \cdots \wedge\left(d_{n-1}-d_{n}\right) \wedge \inf \left\{d_{n}-d_{i}: i>n\right\} \\
& \geq\left(d_{n-1}-d_{n}\right) \wedge\left(d_{n}-d_{n+1}\right) .
\end{aligned}
$$

If $n>n_{2}$ then $\operatorname{gap}\left(X, x_{n}\right) \geq(1-\varrho)\left(d_{n-1} \wedge d_{n}\right)=(1-\varrho) d_{n}$ as desired.

Returning to the proof of Theorem 2.8, we consider two cases according to the value of $\varrho$.

CASE 1 . If $\varrho<1 / 2$, choose $\lambda=1 / \varrho>2$. Then $\gamma_{n}=\min \left\{\varrho^{n-i} \operatorname{gap}\left(X, x_{i}\right)\right.$ : $i=1, \ldots, n\}$. For $n \leq n_{2}$ we have the trivial estimate

$$
\gamma_{n} \geq \frac{\varrho^{n_{2}-1} \varepsilon_{Z}}{\operatorname{diam} X} d_{n}
$$

For $1 \leq i \leq n_{2}<n$ we estimate

$$
\varrho^{n-i} \operatorname{gap}\left(X, x_{i}\right) \geq \frac{\varrho^{n_{2}-i} \varepsilon_{Z}}{d_{n_{2}}} \varrho^{n-n_{2}} d_{n_{2}} \geq \frac{\varrho^{n_{2}-1} \varepsilon_{Z}}{\operatorname{diam} X} d_{n},
$$


while for $n_{2}<i \leq n$ we use Lemma 3.13 to estimate

$$
\varrho^{n-i} \operatorname{gap}\left(X, x_{i}\right) \geq(1-\varrho) \varrho^{n-i} d_{i} \geq(1-\varrho) d_{n} .
$$

In all cases we obtain

$$
\gamma_{n} \geq \min \left\{1-\varrho, \frac{\varrho^{n_{2}-1} \varepsilon_{Z}}{\operatorname{diam} X}\right\} d_{n}
$$

and deduce (3.4) with

$$
\omega_{0}(t)=\max \left\{\frac{1}{1-\varrho}, \frac{\operatorname{diam} X}{\varrho^{n_{2}-1} \varepsilon_{Z}}\right\} t .
$$

CASE 2. If $1 / 2 \leq \varrho<1$, choose $\lambda=3$ and

$$
\alpha=\log (1 / \varrho) / \log 3 \text {. }
$$

Then $\gamma_{n}^{\alpha}=\min \left\{\varrho^{n-i} \operatorname{gap}\left(X, x_{i}\right)^{\alpha}: i=1, \ldots, n\right\}$. For $n \leq n_{2}$ we have the trivial estimate

$$
\gamma_{n}^{\alpha} \geq \frac{\varrho^{n_{2}-1} \varepsilon_{Z}^{\alpha}}{\operatorname{diam} X} d_{n}
$$

As in the preceding case we deduce that

$$
\varrho^{n-i} \operatorname{gap}\left(X, x_{i}\right)^{\alpha} \geq \begin{cases}(\operatorname{diam} X)^{-1} \varrho^{n_{2}-1} \varepsilon_{Z}^{\alpha} d_{n}, & 1 \leq i \leq n_{2}<n \\ (\operatorname{diam} X)^{\alpha-1}(1-\varrho)^{\alpha} d_{n}, & n_{2}<i \leq n\end{cases}
$$

Hence

$$
\gamma_{n}^{\alpha} \geq \min \left\{\frac{(1-\varrho)^{\alpha}}{(\operatorname{diam} X)^{1-\alpha}}, \frac{\varrho^{n_{2}-1} \varepsilon_{Z}^{\alpha}}{\operatorname{diam} X}\right\} d_{n},
$$

so (3.4) holds with

$$
\omega_{0}(t)=\max \left\{\frac{(\operatorname{diam} X)^{1-\alpha}}{(1-\varrho)^{\alpha}}, \frac{\operatorname{diam} X}{\varrho^{n_{2}-1} \varepsilon_{Z}^{\alpha}}\right\} t^{\alpha} .
$$

Using Theorem 3.2, we conclude that $F^{-1}$ is $\alpha$-Hölder continuous if $1 / 2 \leq$ $\varrho<1$, where $\alpha$ is the value in (3.14), and that $F^{-1}$ is Lipschitz continuous if $\varrho<1 / 2$.

3.15. Remark. We do not know whether there exists a space $X$, with $L(X)$ infinite, so that $K(X)$ admits a bi-Lipschitz, or Lipschitz and coHölder, embedding in a finite-dimensional Euclidean space.

\section{Proof of Theorem 2.15}

4.1. Iterated function systems and iterated function complexes. An iterated function system (or IFS) is a finite collection of contractions of a complete metric space. The basic theory of IFS's can be found in standard textbooks of fractal geometry, e.g., [11], [30], [10] or [23]. We briefly recall the basic theory. 
Associated to each IFS $\mathcal{F}$ on a complete metric space $X$ is a unique nonempty compact set $A=A(\mathcal{F}) \in K(X)$, called the attractor of $\mathcal{F}$, characterized by the relation $A=F(A)$, where

$$
F(S):=\bigcup_{f \in \mathcal{F}} f(S)
$$

Indeed, the map $F$ defined in (4.2) is a contraction of $K(X)$, and the attractor $A$ is the unique fixed point for $F$. It is easy to see that $A=\bigcap_{m=1}^{\infty} F^{(m)}(T)$ for any $T \in K(X)$ with $F(T) \subset T$, where $F^{(m)}$ denotes the $m$-fold composition of $F$.

Let $M$ be the cardinality of $\mathcal{F}$. For $m \in \mathbb{N}$, we let $W_{M}^{m}=\{1, \ldots, M\}^{m}$ be the space of words of length $m$ on $M$ letters. We abbreviate $W_{M}:=W_{M}^{1}$. For $m=0$ we set $W_{M}^{0}=\{\emptyset\}$. We let $W_{M}^{*}=\bigcup_{m \geq 0} W_{M}^{m}$ be the space of all finite words, and $\Sigma_{M}=\{1, \ldots, M\}^{\mathbb{N}}$ be the space of infinite words on $M$ letters (commonly referred to as the shift space or symbol space). We denote elements of $W_{M}^{m}, m \in \mathbb{N}$, and $\Sigma_{M}$ by concatenation of letters, i.e., $w=w_{1} \cdots w_{m} \in W_{M}^{m}$ or $w=w_{1} w_{2} \cdots \in \Sigma_{M}$. For $v \in W_{M}^{*}$ and $w \in W_{M}^{*} \cup \Sigma_{M}$ we denote by $v w$ the word obtained by adjoining $v=v_{1} \cdots v_{m}$ to the left of $w=w_{1} w_{2} \cdots$, i.e., $v w=v_{1} \cdots v_{m} w_{1} w_{2} \cdots$. Conversely, for $w \in \Sigma_{M}$ and $m \in \mathbb{N}$ we denote by $[w]_{m}$ the word in $W^{m}$ consisting of the first $m$ letters of $w$; thus $w=[w]_{m} w^{\prime}$ for some $w^{\prime} \in \Sigma_{M}$.

For each IFS $\mathcal{F}=\left\{f_{1}, \ldots, f_{M}\right\}$ there is a canonical surjection $\pi_{\mathcal{F}}: \Sigma_{M} \rightarrow$ $A(\mathcal{F})$ given by

$$
\left\{\pi_{\mathcal{F}}\left(w_{1} w_{2} \cdots\right)\right\}=\bigcap_{m=1}^{\infty} f_{w_{1}} \circ \cdots \circ f_{w_{m}}(T),
$$

where $T$ is any element of $K(X)$ with $F(T) \subset T$. We write $f_{w}=f_{w_{1}} \circ \cdots \circ f_{w_{m}}$ for any $w=w_{1} \cdots w_{m} \in W_{M}^{*}$.

We now introduce a generalization of the notion of IFS.

4.3. Definition. Let $\mathcal{F}_{1}, \ldots, \mathcal{F}_{r}$ be a finite collection of iterated function systems on a complete metric space $X$. For $v=v_{1} v_{2} \cdots \in \Sigma_{r}$, let $\mathcal{F}_{v}=\left(\mathcal{F}_{v_{1}}, \mathcal{F}_{v_{2}}, \ldots\right)$. The collection of sequences $\left\{\mathcal{F}_{v}\right\}_{v \in \Sigma_{r}}$ is called an iterated function complex (of rank $r$ ), or IFC.

Associated to each rank $r$ IFC $\left\{\mathcal{F}_{v}\right\}_{v \in \Sigma_{r}}$ is a family $\left\{A^{v}\right\}_{v \in \Sigma_{r}}$ of nonempty compact subsets of $X$, the attractor of $\left\{\mathcal{F}_{v}\right\}$, characterized by the identity

$$
A^{v}=\bigcap_{m=1}^{\infty} F_{v_{1}} \circ \cdots \circ F_{v_{m}}(T)
$$

for any $T$ such that $F_{i}(T) \subset T$ for all $i$. Here $F_{i}, i=1, \ldots, r$, denotes the set function for $\mathcal{F}_{i}$ defined in (4.2). Indeed, the set functions $F_{1}, \ldots, F_{r}$ form an IFS on $K(X)$ whose attractor $\mathbb{A} \in K(K(X))$ is precisely the collection $\left\{A^{v}\right\}$. 
Observe that the set $A^{\bar{i}}$ defined by (4.4) with $\bar{i}=(i, i, \ldots)$ coincides with the attractor $A^{i}$ for the IFS $\mathcal{F}_{i}$.

4.5. The Laakso graph. In this subsection, we describe the Laakso graph $G$ in further detail, first as a quotient of the product space $[0,1] \times \Sigma_{2}$, and then as the attractor of a self-similar IFS.

We denote by $I_{0}=[0,1]$ the unit interval in $\mathbb{R}$, which we view as the attractor for the IFS $\mathcal{H}=\left\{h_{1}, h_{2}, h_{3}, h_{4}\right\}$, where

$$
h_{i}: \mathbb{R} \rightarrow \mathbb{R}, \quad h_{i}(x)=(x+i-1) / 4 .
$$

The symbolic coding map $\pi_{\mathcal{H}}: \Sigma_{4} \rightarrow I_{0}$ corresponds to the representation of elements of $I_{0}=[0,1]$ in base four. We let $C$ be the $1 / 4$ Cantor set, i.e., the invariant set for the IFS $\left\{h_{1}, h_{4}\right\}$. For $m \in \mathbb{N}$, let

$$
O_{m}:=\bigcup_{j=0}^{4^{m-1}-1}\left((4 j+1) / 4^{m},(4 j+3) / 4^{m}\right) .
$$

The union of the open sets $O_{m}$ is the complement of $C$ in $I_{0}$.

4.8. Definition. The Laakso graph is the space $G=I_{0} \times \Sigma_{2} / \sim$, where $(t, v) \sim\left(t, v^{\prime}\right)$ if and only if $t \in I_{0} \backslash \bigcup\left\{O_{m}: v_{m} \neq v_{m}^{\prime}\right\}$.

4.9. Example. Let $\overline{1}=111 \cdots$ and $\overline{2}=222 \cdots$. Then $(t, \overline{1}) \sim(t, \overline{2})$ if and only if $t \in C$.

We denote the equivalence class of $(t, v)$ in $G$ by $[t, v]$, and equip $G$ with the canonical quotient path metric, defined as follows:

$$
d\left([t, v],\left[t^{\prime}, v^{\prime}\right]\right)=\inf \sum_{j=1}^{k}|t(j)-t(j-1)|,
$$

where the infimum is taken over all finite sequences

$$
(t, v)=(t(0), v(0)),(t(1), v(1)), \ldots,(t(k), v(k))=\left(t^{\prime}, v^{\prime}\right)
$$

satisfying $[t(j), v(j-1)]=[t(j), v(j)]$ for all $j=1, \ldots, k$. (Compare the "wormhole metrics" of [25] or [26].) Then $G$ is a compact geodesic doubling metric space. The maps $\gamma_{v}: I_{0} \rightarrow G, v \in \Sigma_{2}$, given by $\gamma_{v}(t)=[t, v]$ are isometric embeddings, defining an uncountable family of geodesic segments in $G$ joining the two endpoints.

The space $G$ may be viewed as the attractor for a self-similar IFS of cardinality six. To simplify the description, we use an alternate notation for the alphabet. Thus let

$$
U=\{1,(2,1),(2,2),(3,1),(3,2), 4\} .
$$

The spaces $U^{m}, m \in \mathbb{N}, U^{*}$ and $\Sigma_{U}$ of words of length $m$, finite words, and infinite words are defined in the usual manner. 
To each $u \in U$ there corresponds a set $S_{u} \subset G$ which is the image of $G$ under a contractive similarity $g_{u}$ with scale factor $1 / 4$. These firstorder similarity pieces are indicated in Figure 1 . Thus $G$ is the attractor for the IFS $\mathcal{G}=\left\{g_{u}: u \in U\right\}$ with symbol space $\Sigma_{U}$. As usual, we write $g_{u}=g_{u_{1}} \circ \cdots \circ g_{u_{m}}$ and $S_{u}=g_{u}(G)$ for $u=u_{1} \cdots u_{m} \in U^{m}$.

The following diagram commutes:

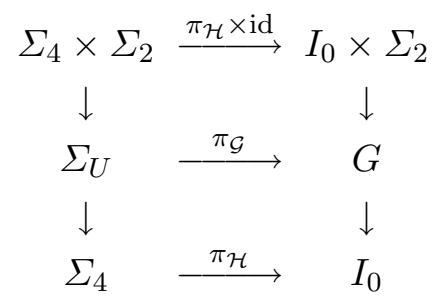

Here the maps from $\Sigma_{4} \times \Sigma_{2}$ to $\Sigma_{U}$ and from $\Sigma_{U}$ to $\Sigma_{4}$ are given, in each coordinate, by the following maps from $W_{4} \times W_{2}$ to $U$ and from $U$ to $W_{4}$ :

$$
\begin{aligned}
(1,1),(1,2) & \rightarrow 1 \rightarrow 1 \\
(2,1) & \rightarrow(2,1) \searrow{ }^{2} \\
(2,2) & \rightarrow(2,2) \nearrow^{2} \\
(3,1) & \rightarrow(3,1) \searrow 3 \\
(3,2) & \rightarrow(3,2) \nearrow^{3} \\
(4,1),(4,2) & \rightarrow 4 \rightarrow 4
\end{aligned}
$$

The map from $I_{0} \times \Sigma_{2}$ to $G$ in (4.10) is the quotient map $(t, v) \mapsto[t, v]$, while the map from $G$ to $I_{0}$ is the projection $[t, v] \mapsto t$. Finally, $\pi_{\mathcal{H}}: \Sigma_{4} \rightarrow I_{0}$ and $\pi_{\mathcal{G}}: \Sigma_{U} \rightarrow G$ denote the symbolic coding maps for the IFS's $\mathcal{H}$ and $\mathcal{G}$.

Sketch of the proof of Theorem 2.15. In Section 4.27, we will construct a specific rank two iterated function complex $\left\{\mathcal{F}^{v}\right\}_{v \in \Sigma_{2}}$ in the plane. The essential feature of the attractor $\left\{A^{v}\right\}_{v \in \Sigma_{2}}$ for this IFC is the equivalence

$$
(t, v) \sim\left(t^{\prime}, v^{\prime}\right) \Leftrightarrow A_{t}^{v}=A_{t}^{v^{\prime}}
$$

which guarantees that the map $\Phi: G \rightarrow K\left(I_{0}\right)$ given by

$$
\Phi([t, v])=A_{t}^{v}
$$

is well defined. We will prove that

$$
\Phi \text { is 33-bi-Lipschitz, }
$$

and

$$
\Phi \circ \gamma_{v}: I_{0} \rightarrow K\left(I_{0}\right) \text { is an isometry for each } v \in \Sigma_{2} .
$$

We now prove Proposition 2.14. Compare the proof in [26, §2.5]. 
Proof of Proposition 2.14. Let $\left(Y, d^{\prime}\right)$ be a round ball space with roundness function $\delta(\varepsilon)$, and suppose that $F: G \rightarrow Y$ is $L$-bi-Lipschitz. For each $x, y \in G$, let $L(x, y):=d^{\prime}(F(x), F(y)) / d(x, y)$ be the Lipschitz constant of $\left.f\right|_{x, y}$. Let $a=S_{1} \cap S_{(2,1)} \cap S_{(2,2)}, b=S_{(3,1)} \cap S_{(3,2)} \cap S_{4}, c=S_{(2,1)} \cap S_{(3,1)}$ and $d=S_{(2,2)} \cap S_{(3,2)}$. Observe that $\{c, d\} \subset B(a, 1 / 4) \cup B(b, 1 / 4)$ and $d(c, d)=1 / 2$. Then $d^{\prime}(F(c), F(d)) \geq L^{-2} d^{\prime}(F(a), F(b))$. From the definition of round ball spaces, we conclude that

$$
\max \{L(a, c), L(a, d), L(c, b), L(d, b)\} \geq\left(1+\delta\left(L^{-2}\right)\right) L(a, b) .
$$

Each pair of points on the left hand side coincides with the endpoints of one of the first-order similarity pieces $S_{u}, u \in U$. Thus the endpoints $x$ and $y$ of one of these pieces satisfy $L(x, y) \geq\left(1+\delta\left(L^{-2}\right)\right) L(a, b)$.

The same argument can be applied to points $a_{u}=S_{u, 1} \cap S_{u,(2,1)} \cap S_{u,(3,1)}$ and $b_{u}=S_{u,(2,2)} \cap S_{u,(3,2)} \cap S_{u, 4}$ for any $u \in U^{m}$ and $m \in \mathbb{N}$. For each such $u$ and $m$ there exists a similarity piece of order $m+1$ with endpoints $x_{u}$ and $y_{u}$ so that $L\left(x_{u}, y_{u}\right) \geq\left(1+\delta\left(L^{-2}\right)\right) L\left(a_{u}, b_{u}\right)$.

Note that $\sum_{m=1}^{\infty} \sum_{u \in\{1,4\}^{m}}\left(b_{u}-a_{u}\right)=1$. Hence $L\left(a_{u}, b_{u}\right) \geq L(0,1)$ for some $u \in\{1,4\}^{m}$, and so

$$
L\left(x_{u^{\prime}}, y_{u^{\prime}}\right) \geq\left(1+\delta\left(L^{-2}\right)\right) L(0,1)
$$

for the endpoints $x_{u^{\prime}}, y_{u^{\prime}}$ of some similarity piece $S_{u^{\prime}}$ of $G$. By self-similarity, $S_{u^{\prime}}$ contains a further similarity piece $S_{u^{\prime} v^{\prime}}$ with endpoints $x_{u^{\prime} v^{\prime}}, y_{u^{\prime} v^{\prime}}$ so that

$$
L\left(x_{u^{\prime} v^{\prime}}, y_{u^{\prime} v^{\prime}}\right) \geq\left(1+\delta\left(L^{-2}\right)\right)^{2} L(0,1) .
$$

Continuing in this fashion, we eventually contradict the $L$-bi-Lipschitz character of $F$.

4.15. A lemma on geodesic segments in $K\left(I_{0}\right)$. The following lemma provides a sufficient condition for the vertical slices of a compact set $A \subset$ $I_{0} \times I_{0}$ to sweep out a geodesic in $K\left(I_{0}\right)$.

We denote by $Q_{0}=I_{0} \times I_{0}$ the unit square in $\mathbb{R}^{2}$. For a compact set $A \subset \mathbb{R}^{2}$, we write $A_{t}=\{x:(t, x) \in A\}$ for the vertical slice of $A$ with abscissa $t$. Finally, for $p=(t, x) \in Q_{0}$, we write

$$
X(p)=\left\{p^{\prime}=\left(t^{\prime}, x^{\prime}\right) \in Q_{0}:\left|t^{\prime}-t\right| \geq\left|x^{\prime}-x\right|\right\} .
$$

Then $X(p)$ is the union of two antipodal sectors with opening angle $\pi / 4$ centered at $p$.

4.16. Lemma. For a compact set $A \subset Q_{0}$, the map $t \mapsto A_{t}$ is an isometric embedding of $I_{0}$ in $K\left(I_{0}\right)$ provided

$$
t \in A_{t} \subset[0, t] \quad \forall t \in I_{0}
$$

and

$$
A \subset \bigcup_{x \in A_{t}} X(t, x) \quad \forall t \in I_{0} .
$$


Condition (4.17) says that $A$ lies in the triangle

$$
T_{0}=\{(t, x): 0 \leq x \leq t \leq 1\}
$$

and contains the diagonal $\{(t, t): 0 \leq t \leq 1\}$, while condition (4.18) says that whenever an open vertical segment $J$ is in the complement of $A$, then the open square with diagonal $J$ is also in the complement of $A$.

Proof of Lemma 4.16. Let $0 \leq t^{\prime}<t \leq 1$. Condition (4.17) implies that

$$
D\left(A_{t}, A_{t^{\prime}}\right) \geq \operatorname{dist}\left(t, A_{t^{\prime}}\right) \geq\left|t-t^{\prime}\right| .
$$

On the other hand, condition (4.18) implies that $\{t\} \times A_{t} \subset \bigcup_{x^{\prime} \in A_{t^{\prime}}} X\left(x^{\prime}, t^{\prime}\right)$ and the corresponding statement with the roles of $(t, x)$ and $\left(t^{\prime}, x^{\prime}\right)$ reversed. Consequently,

$$
D\left(A_{t}, A_{t^{\prime}}\right) \leq\left|t-t^{\prime}\right| .
$$

Inequalities (4.20) and (4.21) show that $t \mapsto A_{t}$ is an isometric embedding.

4.22. Remark. Inequality (4.20) can be generalized as follows: if $A$ and $A^{\prime}$ are compact sets in $Q_{0}$ satisfying (4.17) and (4.18), then $D\left(A_{t}, A_{t^{\prime}}^{\prime}\right) \geq$ $\left|t-t^{\prime}\right|$ for all $t, t^{\prime} \in I_{0}$. The proof is the same.

4.23. Two planar iterated function systems. We begin by defining two planar IFS's corresponding to the digits in the alphabet $W_{2}$.

The 4-adic subsquares of $Q_{0}$ are the sixteen squares $[(i-1) / 4, i / 4] \times$ $[(j-1) / 4, j / 4], i, j=1,2,3,4$.

The IFS $\mathcal{F}_{1}$ consists of nine planar contractive maps. Eight of these maps are contractive similarities, with scale factor $1 / 4$, while the ninth is a degenerate affine contraction, with scale factor $1 / 4$ in one direction and zero in the other direction. These nine contractions are indicated pictorially in the left part of Figure 2; they are the eight contractive maps of $\mathbb{R}^{2}$ which send the ordered triple $A, B, C$ of vertices of $Q_{0}$ to each of the ordered triples $a, b, c$ of vertices of the specified 4-adic subsquares of $Q_{0}$, together with the degenerate map sending $A$ and the pair $B, C$ to the endpoints $a$ and $b$ of the line segment labelled " 8 ". We label these maps $f_{1}^{j}, j=1, \ldots, 9$, as indicated in the left part of Figure 2.

The IFS $\mathcal{F}_{2}$ consists of ten contractive similarities, each with scale factor $1 / 4$. These similarities $f_{2}^{j}, j=1, \ldots, 10$, are indicated pictorially in the right part of Figure 2.

4.24. Remark. Observe that $\mathcal{F}_{2}$ contains two contractions $f_{2}^{5}$ and $f_{2}^{8}$ mapping into a common subsquare. However, both $\mathcal{F}_{1}$ and $\mathcal{F}_{2}$ satisfy the open set condition $[11, \S 9.2]$.

4.25. Remark. Note that the maps in the first and last columns coincide: $f_{1}^{j}=f_{2}^{j}$ for $j=1,4,7$ and $f_{1}^{9}=f_{2}^{10}$. In the middle two columns, 

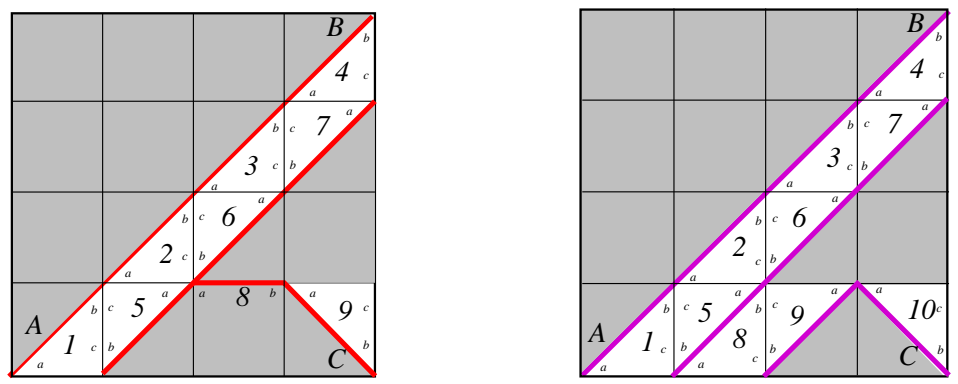

Fig. 2. Similarities comprising $\mathcal{F}_{1}$ (left), $\mathcal{F}_{2}$ (right)

the set of maps is not the same: $\left\{f_{1}^{2}, f_{1}^{5}\right\} \neq\left\{f_{2}^{2}, f_{2}^{5}, f_{2}^{8}\right\}$ and $\left\{f_{1}^{3}, f_{1}^{6}, f_{1}^{8}\right\} \neq$ $\left\{f_{2}^{3}, f_{2}^{6}, f_{2}^{9}\right\}$.

4.26. Remark. Figure 3 shows the attractors $A^{i}$ for the IFS's $\mathcal{F}_{i}, i=$ 1,2. It is clear that conditions (4.17) and (4.18) hold for these attractors. It follows from the previous remark and the self-similarity of the constructions that $A^{1}(m)_{t}=A^{2}(m)_{t}$ if and only if $t \in I_{0} \backslash \bigcup_{j=1}^{m} O_{j}$ for each $m \in \mathbb{N}$, where $A^{i}(m)$ is the image of $T_{0}$ under $m$ iterates of $F^{i}, i=1,2$. Hence $A_{t}^{1}=A_{t}^{2}$ if and only if $t \in C$, as asserted in (4.12) (see Example 4.9).

4.27. A planar iterated function complex. We now consider the rank two IFC $\left\{\mathcal{F}_{v}\right\}_{v \in \Sigma_{2}}$ defined by $\mathcal{F}_{1}$ and $\mathcal{F}_{2}$. To aid the reader, we describe the structure of the attractor of this IFC in greater detail. Given an infinite word $v=v_{1} v_{2} \cdots \in \Sigma_{2}$, let $W_{v}^{m}=W_{v_{1}} \times \cdots \times W_{v_{m}}$ and $f_{v}^{w}=f_{v_{1}}^{w_{1}} \circ \cdots \circ f_{v_{m}}^{w_{m}}$ for all $w \in W_{v}^{m}$, and let

$$
F_{v}^{m}(S):=\bigcup_{w \in W_{v}^{m}} f_{v}^{w}(S)
$$

The attractor for $\left\{\mathcal{F}_{v}\right\}_{v \in \Sigma_{2}}$ is the family $\left\{A^{v}\right\}_{v \in \Sigma_{2}}$ of compact sets, where $A^{v}=\bigcap_{m=1}^{\infty} F_{v}^{m}\left(T_{0}\right)$. When $v=\overline{1}, A^{v}$ coincides with the attractor $A^{1}$ in the left part of Figure 3; when $v=\overline{2}, A^{v}$ coincides with $A^{2}$ in the right
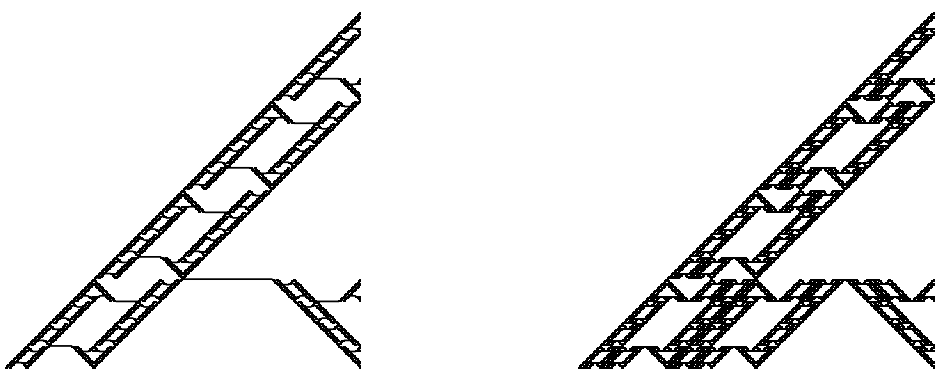

Fig. 3. Attractors $A^{1}$ and $A^{2}$ 
part of Figure 3. For any $v \in \Sigma_{2}, A^{v}$ is the Hausdorff limit of the sequence $A^{v}(m):=F_{v_{1}} \circ \cdots \circ F_{v_{m}}\left(T_{0}\right)$.

We now extend Remark 4.26 to the full IFC.

4.29. Proposition. For each $v \in \Sigma_{2}, A^{v}$ satisfies (4.17) and (4.18). Furthermore, (4.12) holds.

Proof. Since $F_{i}\left(Q_{0}\right) \subset T_{0}$ and $\mathcal{F}_{i} \supset\left\{f_{i}^{j}: j=1,2,3,4\right\}$ for each $i=1,2$, (4.17) holds. To prove (4.18), let $U_{1}, U_{2}$ be the open sets shaded in the left and right parts of Figure 2 respectively. Then

$$
A^{v}(m)=\overline{\operatorname{int}\left(Q_{0}\right) \backslash \bigcup_{i=1}^{m} \bigcup_{w \in W_{v}^{i}} f_{v}^{w}\left(U_{v_{i}}\right)}
$$

for each $m$, where $\operatorname{int}\left(Q_{0}\right)$ denotes the interior of $Q_{0}$ and $\bar{S}$ denotes the closure of a set $S$. It follows that (4.18) is satisfied for each $A^{v}(m), m \in \mathbb{N}$, and hence (4.18) is satisfied for $A^{v}=\bigcap_{m} A^{v}(m)$.

To see why (4.12) holds, observe that Remark 4.25 guarantees that

$$
A^{v}(m)_{t}=A^{v^{\prime}}(m)_{t} \Leftrightarrow t \in I_{0} \backslash \bigcup_{j=1, \ldots, m: v_{j} \neq v_{j}^{\prime}} O_{j}
$$

for all $v, v^{\prime} \in \Sigma_{2}$ and $m \in \mathbb{N}$, where $O_{m}$ is the open set defined in (4.7). Then (4.12) follows by intersecting these conditions over all $m$ and using the definition of the equivalence relation on $I_{0} \times \Sigma_{2}$ (see Definition 4.8).

4.30. Corollary. The map $\Phi: G \rightarrow K\left(I_{0}\right)$ given by $\Phi([t, v])=A_{t}^{v}$ is well defined.

4.31. Subsets and supersets of the sets $A_{t}^{v}$. In this section we prove two technical lemmas describing certain distinguished subsets and supersets of the vertical slices $A_{t}^{v}$. These lemmas will play a key role in the proof of Theorem 2.15 by yielding lower bounds for the Hausdorff distance between slices $A_{t}^{v}$ and $A_{t^{\prime}}^{v^{\prime}}$.

We begin by observing the common elements of the two parts of Figure 2. Figure 4 shows a set of line segments $\Gamma \subset Q_{0}$ which are common to these two figures. Explicitly,

$$
\Gamma=\bigcup_{j=1}^{7} f_{1}^{j}(\Delta) \cup f_{1}^{9}(\Delta)=\bigcup_{j=1}^{8} f_{2}^{j}(\Delta) \cup f_{2}^{10}(\Delta),
$$

where $\Delta:=\left\{(t, t): t \in I_{0}\right\}$ denotes the principal diagonal of $I_{0}$. Thus $\Gamma \subset F_{i}(\Delta)$ for $i=1,2$, whence $\Gamma \subset A^{v}$ for any $v \in \Sigma_{2}$. In fact,

$$
F_{v}^{m}(\Gamma) \subset A^{v}
$$

for all $m \in \mathbb{N}$, where $F_{v}^{m}$ is the set function defined in (4.28). 


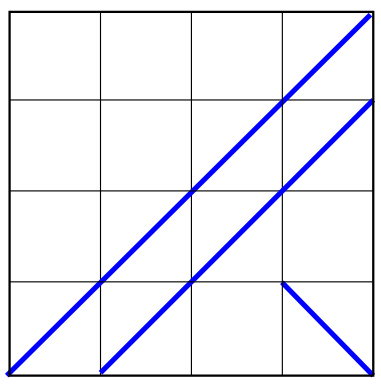

Fig. 4. The set $\Gamma$

Recall our notation $[u]_{m}$ for the element of $U^{m}$ consisting of the first $m$ letters of $u \in \Sigma_{U}$. For each $m \in \mathbb{N}$, the projection of $S_{[u]_{m}} \subset G$ to $I_{0}$ (via the map in (4.10)) is an interval $\left[a_{u, m}, b_{u, m}\right]$ with

$$
b_{u, m}-a_{u, m}=\operatorname{diam} S_{[u]_{m}}=4^{-m} .
$$

If $[t, v]=\pi_{\mathcal{G}}(u)$, then

$$
a_{u, m}+\frac{i\left(u_{m+1}\right)-1}{4} \cdot 4^{-m} \leq t \leq a_{u, m}+\frac{i\left(u_{m+1}\right)}{4} \cdot 4^{-m},
$$

where $i: U \rightarrow W_{4}$ is as in (4.11).

In our first lemma, we describe some subsets of the slices $A_{t}^{v}$. Let $h_{i}$, $i=1,2,3,4$, be the maps from (4.6).

4.35. Lemma. Let $u \in \Sigma_{U}$ with $\pi_{\mathcal{G}}(u)=[t, v]$ and let $m \in \mathbb{N}$.

(i) If $u_{m+1}=(3,2)$, then

$$
A_{t}^{v} \supset h_{i\left(u_{1}\right)} \circ \cdots \circ h_{i\left(u_{m}\right)} \circ h_{1}\left(\Gamma_{s}\right),
$$

where $s=\left(a_{u, m}+\frac{3}{4} \cdot 4^{-m}-t\right) /\left(\frac{1}{4} \cdot 4^{-m}\right)$.

(ii) If $u_{m+1}=(2,2)$, then

$$
A_{t}^{v} \supset h_{i\left(u_{1}\right)} \circ \cdots \circ h_{i\left(u_{m}\right)} \circ h_{1}\left(\widetilde{\Gamma}_{s}\right),
$$

where $s=\left(t-a_{u, m}-\frac{1}{4} \cdot 4^{-m}\right) /\left(\frac{1}{4} \cdot 4^{-m}\right)$ and $\widetilde{\Gamma}=\Gamma \cup f_{1}^{1}(\Gamma) \cup f_{1}^{1} \circ$ $f_{1}^{1}(\Gamma) \cup \cdots$.

To each $u \in U$, we associate a collection $C(u)$ of elements of $\mathcal{F}^{1} \cup \mathcal{F}^{2}$ as follows:

$$
\begin{aligned}
& C(1)=\left\{f_{1}^{1}\right\}, \quad C(2,1)=\left\{f_{1}^{2}, f_{1}^{5}\right\}, \quad C(3,1)=\left\{f_{1}^{3}, f_{1}^{6}, f_{1}^{8}\right\}, \\
& C(2,2)=\left\{f_{2}^{2}, f_{2}^{5}, f_{2}^{8}\right\}, \quad C(3,2)=\left\{f_{2}^{3}, f_{2}^{6}, f_{2}^{9}\right\}, \quad C(4)=\left\{f_{2}^{4}, f_{2}^{7}, f_{2}^{10}\right\} .
\end{aligned}
$$

Observe that each collection $C(u)$ contains precisely one diagonal contraction, namely $H_{i(u)}:=h_{i(u)} \times h_{i(u)}$. (By a diagonal contraction we mean one of the maps $f_{1}^{i}, i=1,2,3,4$.) 
Proof of Lemma 4.35. Let $u \in \Sigma_{U}$ be as in the statement of the lemma. From (4.32), we deduce that

$$
A^{v} \supset H_{i\left(u_{1}\right)} \circ \cdots \circ H_{i\left(u_{m}\right)}\left(F_{v_{m+1}}(\Gamma)\right) .
$$

If $u_{m+1}=(3,2)$, then

$$
A_{t}^{v} \supset\left[H_{i\left(u_{1}\right)} \circ \cdots \circ H_{i\left(u_{m}\right)} \circ f_{2}^{9}(\Gamma)\right]_{t}=h_{i\left(u_{1}\right)} \circ \cdots \circ h_{i\left(u_{m}\right)} \circ h_{1}\left(\Gamma_{s}\right)
$$

where $s=\left(a_{u, m}+\frac{3}{4} \cdot 4^{-m}-t\right) /\left(\frac{1}{4} \cdot 4^{-m}\right)$. (Note that $f_{2}^{9}(t, x)=\left(h_{3}(1-t)\right.$, $\left.h_{1}(1-x)\right)$.)

Similarly, if $u_{m+1}=(2,2)$, then

$$
A_{t}^{v} \supset\left[H_{i\left(u_{1}\right)} \circ \cdots \circ H_{i\left(u_{m}\right)} \circ f_{2}^{8}(\Gamma)\right]_{t}=h_{i\left(u_{1}\right)} \circ \cdots \circ h_{i\left(u_{m}\right)} \circ h_{1}\left(\widetilde{\Gamma}_{s}\right),
$$

where $s=\left(t-a_{u, m}-\frac{1}{4} \cdot 4^{-m}\right) /\left(\frac{1}{4} \cdot 4^{-m}\right)$. (Note that $f_{2}^{8}(t, x)=\left(h_{2}(t), h_{1}(x)\right)$.)

Next, we give some supersets of the slices $A_{t}^{v}$.

4.36. Lemma. Let $u \in \Sigma_{U}$ with $\pi_{\mathcal{G}}(u)=[t, v]$ and let $m \in \mathbb{N}$.

(i) If $u_{m+1}=(3,1)$, then $A_{t}^{v} \subset\left[0, a_{u, m}\right] \cup\left[a_{u, m}+\frac{1}{4} \cdot 4^{-m}, t\right]$.

(ii) If $u_{m+1}=(2,1)$, then $A_{t}^{v} \subset\left[0, a_{u, m}\right] \cup\left[t-\frac{1}{4} \cdot 4^{-m}, t\right]$.

Proof. Let $m \in \mathbb{N}$. By construction,

$$
A^{v}=F_{v}^{m}\left(A^{v}\right) \subset F_{v}^{m}\left(T_{0}\right) .
$$

If $u_{m+1} \in\{(3,1),(2,1)\}$, then $v_{m+1}=1$ and

$$
A^{v} \subset F_{v}^{m}\left(F_{1}\left(T_{0}\right)\right) \subset F_{v}^{m}\left(T_{0} \backslash U_{1}\right),
$$

where $U_{1}$ denotes the open set from the proof of Proposition 4.29. Separating the diagonal and nondiagonal elements gives

$$
A^{v} \subset I_{0} \times\left[0, a_{u, m}\right] \cup H_{i\left(u_{1}\right)} \circ \cdots \circ H_{i\left(u_{m}\right)}\left(T_{0} \backslash U_{1}\right)
$$

whence

$$
\begin{aligned}
A_{t}^{v} & \subset\left[0, a_{u, m}\right] \cup\left[H_{i\left(u_{1}\right)} \circ \cdots \circ H_{i\left(u_{m}\right)}\left(T_{0} \backslash U_{1}\right)\right]_{t} \\
& =\left[0, a_{u, m}\right] \cup h_{i\left(u_{1}\right)} \circ \cdots \circ h_{i\left(u_{m}\right)}\left(\left(T_{0} \backslash U_{1}\right)_{s}\right),
\end{aligned}
$$

where $s=\left(t-a_{u, m}\right) / 4^{-m}$. If $u_{m+1}=(2,1)$, then by $(4.34)$ we have $1 / 4 \leq$ $s \leq 1 / 2$ and $A_{t}^{v} \subset\left[0, a_{u, m}\right] \cup h_{i\left(u_{1}\right)} \circ \cdots \circ h_{i\left(u_{m}\right)}\left(\left[s-\frac{1}{4}, s\right]\right)=\left[0, a_{u, m}\right] \cup\left[t-\frac{1}{4} \cdot 4^{-m}, t\right] ;$ if $u_{m+1}=(3,1)$, then by (4.34) we have $\frac{1}{2} \leq s \leq \frac{3}{4}$ and $A_{t}^{v} \subset\left[0, a_{u, m}\right] \cup h_{i\left(u_{1}\right)} \circ \cdots \circ h_{i\left(u_{m}\right)}\left(\left[\frac{1}{4}, s\right]\right)=\left[0, a_{u, m}\right] \cup\left[a_{u, m}+\frac{1}{4} \cdot 4^{-m}, t\right]$.

Proof of Theorem 2.15. By Corollary 4.30 the map $\Phi: G \rightarrow K\left(I_{0}\right)$ given by $\Phi([t, v])=A_{t}^{v}$ is well defined. The assertion in (4.14) follows from Proposition 4.29. To prove (4.13) we will show that

$$
D\left(A_{t}^{v}, A_{t^{\prime}}^{v^{\prime}}\right) \leq K_{1} d\left(p, p^{\prime}\right)
$$


with $K_{1}=4$, and

$$
d\left(p, p^{\prime}\right) \leq K_{2} D\left(A_{t}^{v}, A_{t^{\prime}}^{v^{\prime}}\right)
$$

with $K_{2}=33$, for all points $p=[t, v]$ and $p^{\prime}=\left[t^{\prime}, v^{\prime}\right]$ in $G$.

4.39. REMARK. We do not know the best bi-Lipschitz constants $K_{1}$ and $K_{2}$ for the embedding $\Phi$. Let $p=[1 / 2,1 v]$ and $p^{\prime}=\left[1 / 2,2 v^{\prime}\right]$ for arbitrary $v, v^{\prime} \in \Sigma_{2}$. Then

$$
\left\{\frac{1}{4}, \frac{1}{2}\right\} \subset \Phi(p)=A_{1 / 2}^{1 v} \subset\left[\frac{1}{4}, \frac{1}{2}\right], \quad\left\{0, \frac{1}{4}, \frac{1}{2}\right\} \subset \Phi\left(p^{\prime}\right)=A_{1 / 2}^{2 v^{\prime}} \subset\left[0, \frac{1}{2}\right],
$$

so $d\left(p, p^{\prime}\right)=1 / 2$ and $D\left(\Phi(p), \Phi\left(p^{\prime}\right)=1 / 4\right.$, which shows that $K_{2}$ cannot be smaller than 2 .

Returning to the proof of the theorem, let $p=[t, v]$ and $p^{\prime}=\left[t^{\prime}, v^{\prime}\right]$ be distinct elements of $G$. Choose a word $[u]_{m} \in U^{m}$ of maximal length $m$ so that $p, p^{\prime} \in S_{[u]_{m}}$. By changing the representatives of $p$ and $p^{\prime}$ if necessary, we may assume that $v_{j}=v_{j}^{\prime}$ for $j=1, \ldots, m$. We begin by noting that

$$
\left|t-t^{\prime}\right| \leq D\left(A_{t}^{v}, A_{t^{\prime}}^{v^{\prime}}\right) \leq \operatorname{diam} S_{[u]_{m}}=4^{-m}
$$

see Remark 4.22 for the lower bound in (4.40).

There exist indices $i, i^{\prime} \in U, i \neq i^{\prime}$, so that $p \in S_{[u]_{m} i}$ and $p^{\prime} \in S_{[u]_{m} i^{\prime}}$. We distinguish several cases according to the values of $i$ and $i^{\prime}$.

CAse 1. Suppose that $\left\{i, i^{\prime}\right\}=\{(2,1),(3,2)\}$ or $\left\{i, i^{\prime}\right\}=\{(2,2),(3,1)\}$. Then

$$
\frac{1}{4} \cdot 4^{-m} \leq d\left(p, p^{\prime}\right) \leq \frac{1}{2} \cdot 4^{-m} .
$$

By (4.40) we see that (4.37) holds with $K_{1}=4$.

If $\left|t-t^{\prime}\right| \geq \frac{1}{8} \cdot 4^{-m}$, then (4.38) holds with $K_{2}=4$ by (4.40) and (4.41). Suppose that $\left|t-t^{\prime}\right| \leq \frac{1}{8} \cdot 4^{-m}$. Let $c_{u, m}=\left(a_{u, m}+b_{u, m}\right) / 2=a_{u, m}+\frac{1}{2} \cdot 4^{-m}$. Then $\left|t-t^{\prime}\right|=\left|t-c_{u, m}\right|+\left|c_{u, m}-t^{\prime}\right|$ and

$$
\begin{aligned}
D\left(A_{t}^{v}, A_{t^{\prime}}^{v^{\prime}}\right) & \geq D\left(A_{c_{u, m}}^{v}, A_{c_{u, m}}^{v^{\prime}}\right)-D\left(A_{t}^{v}, A_{c_{u, m}}^{v}\right)-D\left(A_{c_{u, m}}^{v^{\prime}}, A_{t^{\prime}}^{v^{\prime}}\right) \\
& \geq D\left(A_{c_{u, m}}^{v}, A_{c_{u, m}}^{v^{\prime}}\right)-\left|c_{u, m}-t\right|-\left|c_{u, m}-t^{\prime}\right| \\
& =\frac{1}{4} \cdot 4^{-m}-\left|t-t^{\prime}\right|>\frac{1}{8} \cdot 4^{-m}
\end{aligned}
$$

where we used Lemma 4.16 and Remark 4.39 in the second and third lines, respectively. Combining this with (4.40), we see that (4.38) holds with $K_{2}=4$.

We divide the remaining possibilities into two cases:

CASE 2. $\left\{i, i^{\prime}\right\} \cap\{1,4\} \neq \emptyset$ or $\left\{i, i^{\prime}\right\}=\{(2,1),(3,1)\}$ or $\left\{i, i^{\prime}\right\}=\{(2,2),(3,2)\}$.

CAse 3. (a) $\left\{i, i^{\prime}\right\}=\{(3,1),(3,2)\}$ or (b) $\left\{i, i^{\prime}\right\}=\{(2,1),(2,2)\}$. 
In Case 2 we may assume that $t<t^{\prime}$ and choose

$$
t^{\prime \prime} \in\left\{a_{u, m}+\frac{1}{4} \cdot 4^{-m}, a_{u, m}+\frac{1}{2} \cdot 4^{-m}, a_{u, m}+\frac{3}{4} \cdot 4^{-m}\right\}
$$

so that $t \leq t^{\prime \prime} \leq t^{\prime}$. In Case 3 (a) we choose $t^{\prime \prime}=a_{u, m}+\frac{1}{4} \cdot 4^{-m}$, while in Case 3(b) we choose $t^{\prime \prime}=a_{u, m}+\frac{3}{4} \cdot 4^{-m}$. In all cases, for a suitable choice of $p^{\prime \prime}=\left[t^{\prime \prime}, v^{\prime \prime}\right]$, we have $\left(t^{\prime \prime}, v\right) \sim\left(t^{\prime \prime}, v^{\prime}\right) \sim\left(t^{\prime \prime}, v^{\prime \prime}\right)$ and

$$
\begin{aligned}
d\left(p, p^{\prime}\right) & =d\left(p, p^{\prime \prime}\right)+d\left(p^{\prime \prime}, p^{\prime}\right) \\
& =\left|t-t^{\prime \prime}\right|+\left|t^{\prime \prime}-t^{\prime}\right|=D\left(A_{t}^{v}, A_{t^{\prime \prime}}^{v^{\prime \prime}}\right)+D\left(A_{t^{\prime \prime}}^{v^{\prime \prime}}, A_{t^{\prime}}^{v^{\prime}}\right) \\
& \geq D\left(A_{t}^{v}, A_{t^{\prime}}^{v^{\prime}}\right)
\end{aligned}
$$

by (4.14) and Lemma 4.16. Thus (4.37) holds with $K_{1}=1$.

The proof of (4.38) splits according to the various cases.

In Case 2, since $t<t^{\prime \prime}<t^{\prime}$, we deduce from (4.42) and (4.40) that $d\left(p, p^{\prime}\right)=\left|t-t^{\prime}\right| \leq D\left(A_{t}^{v}, A_{t^{\prime}}^{v^{\prime}}\right)$. Thus (4.38) holds with $K_{2}=1$ in this case.

In Case 3(a), we have $a_{u, m}+\frac{1}{2} \cdot 4^{-m} \leq t, t^{\prime} \leq t^{\prime \prime}=a_{u, m}+\frac{3}{4} \cdot 4^{-m}$. We may assume that $i=(3,1)$ and $i^{\prime}=(3,2)$. By Lemma 4.35(i), $A_{t^{\prime}}^{v^{\prime}}$ contains a point $x^{\prime}$ such that

$$
a_{u, m}+\frac{1}{16} \cdot 4^{-m} \leq x^{\prime} \leq \max \left\{a_{u, m}+\frac{1}{8} \cdot 4^{-m}, t^{\prime}-\frac{1}{2} \cdot 4^{-m}\right\} ;
$$

Lemma 4.36(i) guarantees that $A_{t}^{v} \subset\left[0, a_{u, m}\right] \cup\left[a_{u, m}+\frac{1}{4} \cdot 4^{-m}, t\right]$. Hence

$$
\begin{aligned}
D\left(A_{t}^{v}, A_{t^{\prime}}^{v^{\prime}}\right) & \geq \min \left\{x^{\prime}-a_{u, m}, a_{u, m}+\frac{1}{4} \cdot 4^{-m}-x^{\prime}\right\} \\
& \geq \min \left\{\frac{1}{16} \cdot 4^{-m}, t^{\prime \prime}-t^{\prime}\right\} \geq \frac{1}{4}\left(t^{\prime \prime}-t^{\prime}\right)
\end{aligned}
$$

since $t^{\prime \prime}-t^{\prime} \leq \frac{1}{4} \cdot 4^{-m}$. Thus

$$
d\left(p, p^{\prime}\right)=2 t^{\prime \prime}-t-t^{\prime} \leq 2\left(t^{\prime \prime}-t^{\prime}\right)+\left|t^{\prime}-t\right| \leq 9 D\left(A_{t}^{v}, A_{t^{\prime}}^{v^{\prime}}\right)
$$

by the first two equalities of (4.42) and by (4.40). We obtain (4.38) with $K_{2}=9$.

In Case 3(b), we have $t^{\prime \prime}=a_{u, m}+\frac{1}{4} \cdot 4^{-m} \leq t, t^{\prime} \leq a_{u, m}+\frac{1}{2} \cdot 4^{-m}$. We may assume that $i=(2,1)$ and $i^{\prime}=(2,2)$. If $t-t^{\prime \prime} \leq \frac{15}{16}\left(t^{\prime}-t^{\prime \prime}\right)$, then

$$
D\left(A_{t}^{v}, A_{t^{\prime}}^{v^{\prime}}\right) \geq\left|t-t^{\prime}\right| \geq \frac{1}{16}\left(t^{\prime}-t^{\prime \prime}\right) .
$$

Otherwise, choose an integer $k \geq 1$ so that

$$
t^{\prime \prime}+\frac{1}{4^{k}} \cdot 4^{-m} \leq t^{\prime} \leq t^{\prime \prime}+\frac{1}{4^{k-1}} \cdot 4^{-m} .
$$

By Lemma 4.35(ii), $A_{t^{\prime}}^{v^{\prime}}$ contains a point $x^{\prime}$ such that

$$
a_{u, m}+\frac{1}{4^{k+1}} \cdot 4^{-m} \leq x^{\prime} \leq t^{\prime}-\left(\frac{1}{4}+\frac{3}{4^{k+1}}\right) 4^{-m} .
$$


Next, Lemma 4.36(ii) guarantees that $A_{t}^{v} \subset\left[0, a_{u, m}\right] \cup\left[t-\frac{1}{4} \cdot 4^{-m}, t\right]$. Hence

$$
\begin{aligned}
D\left(A_{t}^{v}, A_{t^{\prime}}^{v^{\prime}}\right) & \geq \min \left\{x^{\prime}-a_{u, m}, t-\frac{1}{4} \cdot 4^{-m}-x^{\prime}\right\} \\
& \geq \min \left\{\frac{1}{4^{k+1}} \cdot 4^{-m}, \frac{3}{4^{k+1}} \cdot 4^{-m}-\left|t-t^{\prime}\right|\right\} \\
& \geq \min \left\{\frac{1}{4^{k+1}} \cdot 4^{-m}, \frac{3}{4^{k+1}} \cdot 4^{-m}-\frac{1}{16}\left(t^{\prime}-t^{\prime \prime}\right)\right\} \\
& \geq \frac{1}{4^{k+1}} \cdot 4^{-m} \geq \frac{1}{16}\left(t^{\prime}-t^{\prime \prime}\right) .
\end{aligned}
$$

In either case, we find $D\left(A_{t}^{v}, A_{t^{\prime}}^{v^{\prime}}\right) \geq \frac{1}{16}\left(t^{\prime}-t^{\prime \prime}\right)$, whence

$$
d\left(p, p^{\prime}\right)=t+t^{\prime}-2 t^{\prime \prime} \leq 2\left(t^{\prime}-t^{\prime \prime}\right)+\left|t^{\prime}-t\right| \leq 33 D\left(A_{t}^{v}, A_{t^{\prime}}^{v^{\prime}}\right)
$$

by the first two equalities of (4.42) and (4.40). We obtain (4.38) with $K_{2}=33$.

\section{Questions and remarks}

Question 1. Does $K([0,1])$ admit a bi-Lipschitz embedding in $L^{1}$ ?

The map $F: K([0,1]) \rightarrow L^{1}([0,1])$ given by $F(A)=\operatorname{dist}(x, A)$ is a 1-Lipschitz embedding. Its inverse can be no better than $1 / 2$-Hölder, as can be seen by considering the distance from an arbitrary point $A \in K(X)$ to $B=[0,1]$. Is $F^{-1} 1 / 2$-Hölder continuous?

QUESTION 2. Is there a constant $L<\infty$ so that every series-parallel graph of diameter one admits an $L$-bi-Lipschitz embedding in $K([0,1])$ ?

As previously observed, Gupta et al. [18] have recently shown that every series-parallel graph admits a 14-bi-Lipschitz embedding in $L^{1}$. Bi-Lipschitz embeddings of graphs and/or finite metric spaces in "good" targets play an increasingly important role in algorithmic problems in computer science and sorting problems (see [18] for a more complete list of applications). It would be interesting to know whether hyperspaces can play the role of the target in embedding results of this type.

Question 3. For which spaces $X$ does $K(X)$ admit a bi-Lipschitz embedding into a uniformly convex Banach space? into a finite-dimensional Euclidean space?

Taking into account the principal theorems of this paper, it is natural to begin an attack on the first part of Question 3 by considering nonuniformly disconnected subsets of $[0,1]$, for example, the "fat" Cantor sets of [6]. When do the hyperspaces of such sets admit bi-Lipschitz embeddings in uniformly convex Banach spaces? For the second part of Question 3, see Remark 3.15. 
Other classes of hyperspaces. For a fixed closed set $C$ in a metric space $X$, denote by $K(X, C)$ the hyperspace of all closed subsets $A \subset X$ with $D(A, C)<\infty$. Thus $K\left(X,\left\{x_{0}\right\}\right)$ (for fixed $\left.x_{0} \in X\right)$ is the hyperspace of all closed and bounded sets in $X$, which coincides with $K(X)$ when $X$ is a proper (or Heine-Borel) metric space. The metric structure of hyperspaces of the type $K(X, C)$ is considered by Kovalev and the author in [24]. Here is a sample result from that study related to the topic of this paper: For any connected metric space $X$ and unbounded closed set $C \subset X$, the hyperspace $K(X, C)$ contains an isometric copy of $\ell_{+}^{\infty}=\left\{x=\left(x_{k}\right) \in \ell^{\infty}: x_{k} \geq 0\right.$ for all $k\}$ and hence of every bounded separable metric space. Furthermore, $K(X, C)$ contains a $\sqrt{2}$-bi-Lipschitz embedded copy of every separable metric space.

\section{References}

[1] M. Aschbacher, P. Baldi, E. B. Baum and R. M. Wilson, Embeddings of ultrametric spaces in finite-dimensional structures, SIAM J. Algebraic Discrete Methods 8 (1987), 564-577.

[2] P. Assouad, Plongements lipschitziens dans $\mathbb{R}^{n}$, Bull. Soc. Math. France 111 (1983), 429-448.

[3] C. Bandt, On the metric structure of hyperspaces with Hausdorff metric, Math. Nachr. 129 (1986), 175-183.

[4] L. M. Blumenthal, Theory and Applications of Distance Geometry, 2nd ed., Chelsea, New York, 1970.

[5] E. Boardman, Some Hausdorff measure properties of the space of compact subsets of [0,1], Quart. J. Math. Oxford Ser. (2) 24 (1973), 333-341.

[6] S. M. Buckley, B. Hanson and P. MacManus, Doubling for general sets, Math. Scand. 88 (2001), 229-245.

[7] D. W. Curtis and R. M. Schori, Hyperspaces of Peano continua are Hilbert cubes, Fund. Math. 101 (1978), 19-38.

[8] G. David and S. Semmes, Strong $A_{\infty}$ weights, Sobolev inequalities and quasiconformal mappings, in: Analysis and Partial Differential Equations, Lecture Notes in Pure and Appl. Math. 122, Dekker, New York, 1990, 101-111.

[9] -, - Fractured Fractals and Broken Dreams. Self-Similar Geometry through Metric and Measure, Oxford Lecture Ser. Math. Appl, 7, Oxford Univ. Press, Oxford, 1997.

[10] G. A. Edgar, Integral, Probability, and Fractal Measures, Springer, New York, 1998.

[11] K. J. Falconer, Fractal Geometry. Mathematical Foundations and Applications, Wiley, Chichester, 1990.

[12] P. R. Goodey, Generalized Hausdorff dimension, Mathematika 17 (1970), 324-327.

[13] - Some results concerning the dimension of the space of compact subsets of the unit interval, Quart. J. Math. Oxford Ser. (2) 27 (1976), 467-473.

[14] - Hausdorff measure functions in the space of compact subsets of the unit interval, Trans. Amer. Math. Soc. 226 (1977), 203-208.

[15] P. M. Gruber, The space of compact subsets of $E^{d}$, Geom. Dedicata 9 (1980), 87-90. 
[16] P. M. Gruber and G. Lettl, Isometries of the space of compact subsets of $E^{d}$, Studia Sci. Math. Hungar. 14 (1979), 169-181.

[17] P. M. Gruber and R. Tichy, Isometries of spaces of compact or compact convex subsets of metric manifolds, Monatsh. Math. 93 (1982), 117-126.

[18] A. Gupta, I. Newman, Y. Rabinovich and A. Sinclair, Cuts, trees and $l_{1}$-embeddings of graphs, Combinatorica 24 (2004), 233-269.

[19] J. Heinonen and S. Semmes, Thirty-three yes or no questions about mappings, measures, and metrics, Conform. Geom. Dyn. 1 (1997), 1-12.

[20] A. Hohti, On Lipschitz homogeneity of the Hilbert cube, Trans. Amer. Math. Soc. 291 (1985), 75-86.

[21] J. E. Hutchinson, Fractals and self-similarity, Indiana Univ. Math. J. 30 (1981), $713-747$.

[22] A. Illanes and S. B. Nadler, Jr., Hyperspaces. Fundamentals and Recent Advances, Monogr. Textbooks Pure Appl. Math. 216, Dekker, New York, 1999.

[23] J. Kigami, Analysis on Fractals, Cambridge Tracts in Math. 143, Cambridge Univ. Press, Cambridge, 2001.

[24] L. Kovalev and J. T. Tyson, Metric structure of hyperspaces, in preparation.

[25] T. J. Laakso, Ahlfors $Q$-regular spaces with arbitrary $Q>1$ admitting weak Poincaré inequality, Geom. Funct. Anal. 10 (2000), 111-123.

[26] -, Plane with $A_{\infty}$-weighted metric not bi-Lipschitz embeddable to $\mathbb{R}_{N}$, Bull. London Math. Soc. 34 (2002), 667-676.

[27] U. Lang and C. Plaut, Bilipschitz embeddings of metric spaces into space forms, Geom. Dedicata 87 (2001), 285-307.

[28] J. R. Lee, M. Mendel and A. Naor, Metric structures in $L_{1}$ : dimension, snowflakes and average distortion, European J. Combin., to appear.

[29] A. J. Lemin, Isometric embedding of ultrametric (non-Archimedean) spaces in Hilbert space and Lebesgue space, in: p-Adic Functional Analysis (Ioannina, 2000), Lecture Notes in Pure and Appl. Math. 222, Dekker, New York, 2001, 203-218.

[30] P. Mattila, Geometry of Sets and Measures in Euclidean Spaces, Cambridge Stud. Adv. Math. 44, Cambridge Univ. Press, Cambridge, 1995.

[31] M. McClure, The Hausdorff dimension of the hyperspace of compact sets, Real Anal. Exchange 22 (1996/97), 611-625.

[32] P. A. P. Moran, Additive functions of intervals and Hausdorff measure, Proc. Cambridge Philos. Soc. 42 (1946), 15-23.

[33] A. Pełczyński, A remark on spaces $2^{X}$ for zero-dimensional X, Bull. Acad. Polon. Sci. Sér. Sci. Math. Astronom. Phys. 13 (1965), 85-89.

[34] H. Reiter and A. Reiter, The space of closed subsets of a convergent sequence, Math. Mag. 69 (1996), 217-221.

[35] I. J. Schoenberg, Metric spaces and completely monotone functions, Ann. of Math. (2) 39 (1938), 811-841.

[36] -, Metric spaces and positive definite functions, Trans. Amer. Math. Soc. 44 (1938), $522-536$.

[37] R. M. Schori and J. E. West, Hyperspaces of graphs are Hilbert cubes, Pacific J. Math. 53 (1974), 239-251.

[38] - - - The hyperspace of the closed unit interval is a Hilbert cube, Trans. Amer. Math. Soc. 213 (1975), 217-235.

[39] A. F. Timan, The isometric mapping of certain ultrametric spaces into the $L_{p}$ spaces, Trudy Mat. Inst. Steklov. 134 (1975), 314-326, 411 (in Russian).

[40] J. T. Tyson and J.-M. Wu, Characterizations of snowflake metric spaces, Ann. Acad. Sci. Fenn. Ser. A I Math. 30 (2005), 313-336. 
[41] J. Väisälä, Lipschitz homeomorphisms of the Hilbert cube, Topology Appl. 11 (1980), $103-110$.

Department of Mathematics

University of Illinois

1409 West Green Street

Urbana, IL 61801, U.S.A.

E-mail: tyson@math.uiuc.edu

Received 24 February 2005;

in revised form 20 September 2005 